\title{
Transcranial management of pituitary tumours with suprasellar extension
}

\author{
L I N D A Y S Y M O N A D J A N J A K UBOW S I \\ From the Department of Neurosurgical Studies, The National Hospital, Queen Square, London
}

SUMMARY A consecutive series of 101 pituitary tumours treated in the 10 year period 1968-78 has been examined, giant lesions being excluded. There were 48 female cases and 53 male, women predominating in the ratio of three to two in the age group 40-50 years and men showing a slight predominance in the age group 50-60 years. Most cases presented with visual deterioration which in 22 cases had been present for between one and two years, and in a further 22 for an even longer period, between two and 10 years. All patients underwent subfrontal craniotomy with mainly radical excision of the tumour followed by radiotherapy. The operative mortality was $0.99 \%$. A system of grading of visual field defect has been described and used to compare preoperative visual loss with postoperative visual recovery. Fifty-six per cent of cases returned to normal vision over the first two years, and a further $37 \%$ showed appreciable improvement in visual fields or acuity or both. Six per cent of cases showed no improvement in visual fields, and one patient died of postoperative deep vein thrombosis and pulmonary embolism. The degree of visual improvement has been correlated with the extent of visual defect, length of visual complaint, and size of the tumour. The importance of central and peripheral visual field analysis is emphasised yet again.

Recent improvements in diagnostic, surgical, and radiotherapeutic techniques and in medical management have had considerable general impact on neurosurgical treatment. The management of the patient with pituitary tumour and visual impairment, however, remains an important facet of neurosurgery. The lesion, although usually benign in nature, lies adjacent to important and delicate structures-the optic nerves, major vessels, and hypothalamus. In addition to the potential surgical problems there may be complications of visual complex and endocrinological function.

Reported statistics from operative mortality in management of pituitary adenomas varies in many series and some results have only historical value (Table 1). Postoperative mortality was greatly reduced but not eliminated by the introduction of steroids. Another important predictive factor, first pointed out by $G$. Jefferson (1940) and stressed by Bakay (1950) is the size of the suprasellar extension. Various ways to determine suitability for surgery have been attempted. A. Jefferson (1969)

Address for reprint requests: Mr Jan Jakubowski, Department of Neurosurgical Studies, The National Hospital, Queen Square, London WC1N 3BG.

Accepted 10 August 1978 pointed out the importance both of the extent of the suprasellar extension and the distance from the foramen of Monro to the highest point of the tumour extension. At that time he introduced an index, defined as the ratio of the area of the suprasellar extension on midline tomography to the distance from the foramen of Monro to the tumour.

Many patients show encouraging postoperative visual recovery but in a considerable number of cases vision does not return to normal. Various factors influencing recovery have been postulated, in particular disturbance or asymmetry of vascular supply to the chiasm (Dawson, 1958; Morello and Frera, 1966). An additional difficulty is that although much has been written, there is no simple and comprehensive way of determining overall visual change. A. Jefferson (1957) used a scale system, others have relied on descriptive evaluation.

The importance of postoperative radiation in preventing recurrence has not been uniformly accepted. Arumugasany et al. (1971), Ray and Patterson (1971), and Wirth et al. (1974) strongly recommend postoperative $\mathrm{x}$-ray therapy; others (Stern and Batzdorf, 1970; Kunicki et al., 1975), 
Table 1 Results of studies of operative mortality in cases of pituitary adenoma

\begin{tabular}{|c|c|c|c|}
\hline Author & $\begin{array}{l}\text { Number of } \\
\text { cases }\end{array}$ & $\begin{array}{l}\text { Number of } \\
\text { operations }\end{array}$ & $\begin{array}{l}\text { Percentage of opera- } \\
\text { tive mortality }\end{array}$ \\
\hline $\begin{array}{l}\text { Bakay (Olivecrona) } \\
\text { (1950) }\end{array}$ & 232 & 一 & $\begin{array}{l}35 \% \text { with suprasellar } \\
\text { extension } \\
6.4 \% \text { without supra- } \\
\text { sellar extension }\end{array}$ \\
\hline Horrax et al, (1952) & 125 & 113 & $14.1 \%$ \\
\hline Tönnis et al. (1953) & 264 & - & $10.4 \%$ \\
\hline Mogensen (1957) & 60 & 66 & $12.4 \%$ \\
\hline Rand (1957) & 67 & 85 & $8.9 \%$ \\
\hline $\begin{array}{l}\text { Guillaume and Caron } \\
\text { (1958) }\end{array}$ & - & 141 & $12.0 \%$ \\
\hline Baker (1960) & 150 & 一 & $5.3 \%$ \\
\hline Krayenbühl (1961) & 151 & 一 & $8.6 \%$ \\
\hline Obrador (1961) & - & 65 & $20 \%$ \\
\hline $\begin{array}{l}\text { Elkington and } \\
\text { McKissock (1967) }\end{array}$ & 260 & - & $10.0 \%$ \\
\hline $\begin{array}{l}\text { Stern and Batzdorf } \\
\text { (1970) }\end{array}$ & 64 & 68 & $5.9 \%$ \\
\hline $\begin{array}{l}\text { Ray and Patterson } \\
\text { (1971) }\end{array}$ & 146 & 165 & $\begin{array}{l}1.2 \% \text { (chromophobe } \\
\text { only) }\end{array}$ \\
\hline $\begin{array}{l}\text { Arumugasany et al. } \\
\text { (1971) }\end{array}$ & 66 & - & $3.6 \%$ \\
\hline Kunc (1973) & 263 & 300 & $12.9 \%$ \\
\hline Wirth et al (1974) & 179 & 199 & $6.9 \%$ \\
\hline Kunicki et al. (1975) & 114 & - & $4.8 \%$ \\
\hline Jefferson A. (1978) & 62 & 一 & $\begin{array}{l}3.2 \% \text { (massive lesion } \\
\text { excluded) }\end{array}$ \\
\hline $\begin{array}{l}\text { Symon and } \\
\text { Jakubowski }\end{array}$ & 101 & 101 & $\begin{array}{l}0.99 \% \text { (giant lesions } \\
\text { excluded) }\end{array}$ \\
\hline (present series) & 117 & 117 & $\begin{array}{l}3.4 \% \text { (giant lesions } \\
\text { included) }\end{array}$ \\
\hline
\end{tabular}

have relied more on radical excision of the tumour and natural immunological defence mechanisms than on postoperative radiation.

Most published series consist of cases collected over a long period with variation in diagnostic facilities, surgical technique and radiation methods.

In this paper we present results collected over the last 10 years when the same diagnostic, operative, anaesthetic, and radiological techniques were used by the same team.

\section{Patients and methods}

The material consists of a consecutive series of 101 cases of pituitary adenomas during the period 1968 until 1978. Excluded from the present series have been: (1) two patients with diagnosed pituitary fossa tumours who did not consent to the operation, (2) six patients with recurrent pituitary tumours who were originally operated on elsewhere, and (3) 16 patients with "giant" pituitary tumours selected on the basis of surgical findings and radiological evidence of suprasellar extension over $40 \mathrm{~mm}$ above the planum sphenoidale, less than $6 \mathrm{~mm}$ distance from the highest point of the tumour to the foramen of Monro, or multi- directional spread. Such tumours present a completely different neurosurgical problem and form the subject of a separate study.

Most patients (around $87 \%$ ) were referred to us by neurological colleagues and only $13 \%$ were referred for surgery directly. All cases referred were treated surgically. All patients were operated upon by the senior author with one exception.

Of the 101 patients 48 were women and 53 men. The youngest patient was aged 22 years (female) and the oldest 78 years (female). Sex and age distributions are shown in Fig. 1. Most cases (73) were between 40 and 60 years of age. In the age group 40 to 50 years, women predominated $(21: 14)$ while in the 50 to 60 years age group men predominated $(22: 16)$.

Ninety-four patients complained of visual deterioration. Forty-seven patients complained of hormonal disorders such as amenorrhoea, impotence, loss of libido, tiredness, loss of hair, obesity, polyuria, or galactorrhoea. Headache was a feature in 38 cases. Other neurological abnormalities such as diplopia, upper motor neurone weakness, involuntary tremor, and epilepsy were present in 11 patients, and six patients gave a history of pituitaryo apoplexy (Table 2). The first symptom was visual disturbance in 69 cases, headache in 17 cases, and hormonal disorders in 13 cases. In two cases the first complaint was epilepsy and upper motor neurone type weakness respectively (Table 3 ). Theo occurrence of visual complaint in relation to the first symptom was also analysed. Visual complaintso could appear at any time after the first symptoms or not at all. However, in patients with a long history - that is, exceeding eight years - they might occur very late. Thus careful assessment of the visual condition of such patients should not be abandoned after a few years but should be carried on at regular intervals. Analysis of the duration of

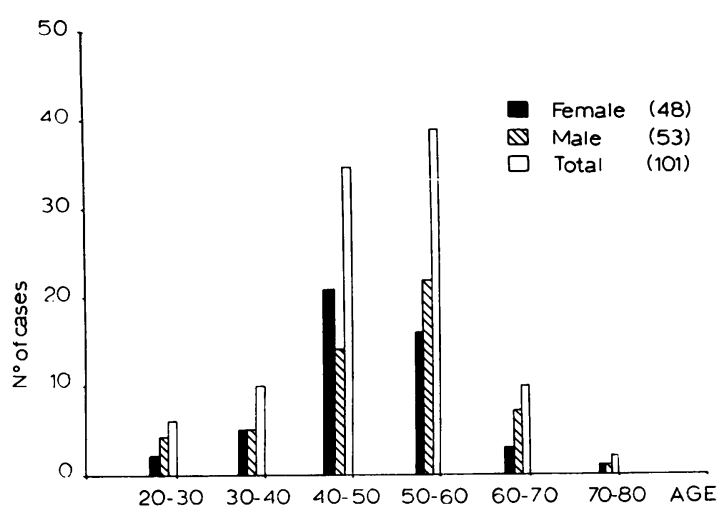

Fig. 1 Age and sex distribution of study sample. 
Table 2 Patients' complaints

\begin{tabular}{lcr}
\hline Complaint & $\begin{array}{l}\text { Number of } \\
\text { cases }\end{array}$ & $\%$ \\
\hline Visual deficits & 94 & 93.1 \\
Headache & 38 & 37.6 \\
Acromegaly & 6 & 5.9 \\
Cushing's (after adrenalectomy) & 3 \\
Other hormonal & 38 & 2.9 \\
Apoplexy & 6 & 37.6 \\
Other neurological & 11 & 5.9 \\
& & 10.0 \\
\hline
\end{tabular}

Table 3 First symptom

\begin{tabular}{lcc}
\hline Symptom & Number of cases & $\%$ \\
\hline Visual & 69 & 68.4 \\
Headache & 17 & 16.8 \\
Hormonal & 13 & 12.9 \\
Other neurological & 2 & 1.9 \\
Total & 101 & 100 \\
\hline
\end{tabular}

visual disturbance (Table 4) showed that only 50 cases had a relatively short history (up to one year), 22 such cases had a moderately long period of visual complaint (one to two years) while the remaining 22 cases had what appeared to be an unacceptably long period of visual complaint, varying between two and 10 years. There was no correlation between the age of the patient and the length of the history of visual disturbance. However, it is worth mentioning here that the highest number of patients with a long history of visual disturbance was in the 40 to 50 years age group $(50 \%)$ and that in ali young people (between 20 and 30 years) the history of visual disturbance was short.

\section{PHYSICAL EXAMINATION}

Of a total of 101 patients, 83 were in a good general condition, 14 patients in a satisfactory condition, and four patients in a generally poor condition. All classified in the latter two groups were either advanced in age or suffering from high blood pressure or cardiac and respiratory illness.

To evaluate visual defects, we developed a point scoring system where each eye was assessed individually according to the state of visual fields and

Table 4 Duration of visual disturbance

\begin{tabular}{lll}
\hline $\begin{array}{l}\text { Length of history } \\
(y r)\end{array}$ & Overall history & History of visual disturbance \\
\hline $0-1$ & 32 & 50 \\
$1-2$ & 24 & 22 \\
$2-4$ & 21 & 15 \\
$4-6$ & 10 & 5 \\
$6-10$ & 5 & 2 \\
$10>$ & 9 & 0 \\
Total & 101 & 94 \\
\hline
\end{tabular}

the visual acuity. The points were then added together and a percentage of visual loss calculated. The scoring and percentages are shown in Table 5 . We attributed $60 \%$ visual loss to isolated uniocular blindness, to take into consideration the loss of stereoscopic vision. All of our cases were evaluated accordingly. In 100 cases there was detectable visual loss (although six patients had no history of visual complaint). One patient had no visual defect and was referred to us because of amenorrhoea, galactorrhoea, and headaches. The distribution of patients according to the degree of visual loss is shown in Table 6. Most patients had a moderate visual deficit $(20$ to $60 \%)$. Ten cases had severe visual loss, $60 \%$ and above, and 11 patients had a minor degree of visual loss, below $20 \%$. We were not able to establish a significant correlation between the age of the patient, percentage of visual loss, and duration of visual disturbance. However, in some cases where rapid worsening of vision occurred, time played an important role.

Further neurological examination revealed 11 patients with weakness of various nerves supplying the ocular muscles. In all but two cases there was radiological and intraoperative evidence of either gross bony destruction around the sella or a large suprasellar mass with a lateral or posterior extension. Six patients had a weakness of the facial muscles of upper motor neurone type, five patients had pyramidal tract signs, and three patients had a tremor of the extrapyramidal type.

Signs of endocrinological disturbance were detected in 66 cases. Those were mainly hypopituitarism; however three patients had diabetes insipidus and another three suffered from Cushing's disease and had undergone adrenalectomy in the past,

Table 5 Scoring points and percentages of visual loss

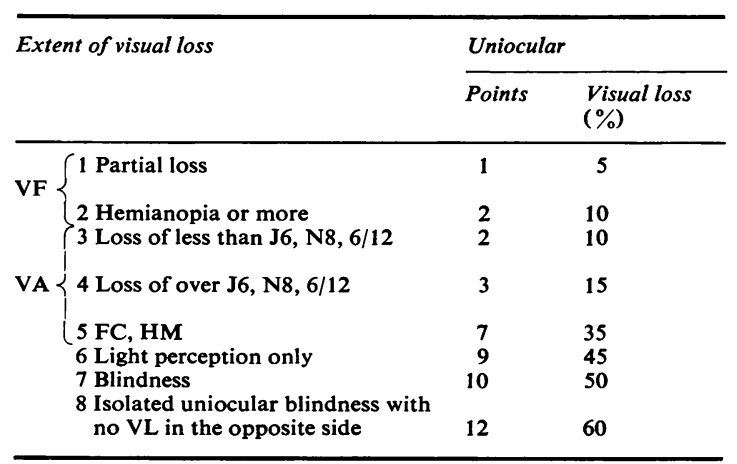

VL $=$ visual loss;

$\mathrm{VF}=$ visual fields;

VA = visual acuity;

$\mathrm{FC}=$ finger counting;

HM $=$ hand movements. 
Table 6 Percentage of preoperative visual loss and postoperative visual recovery

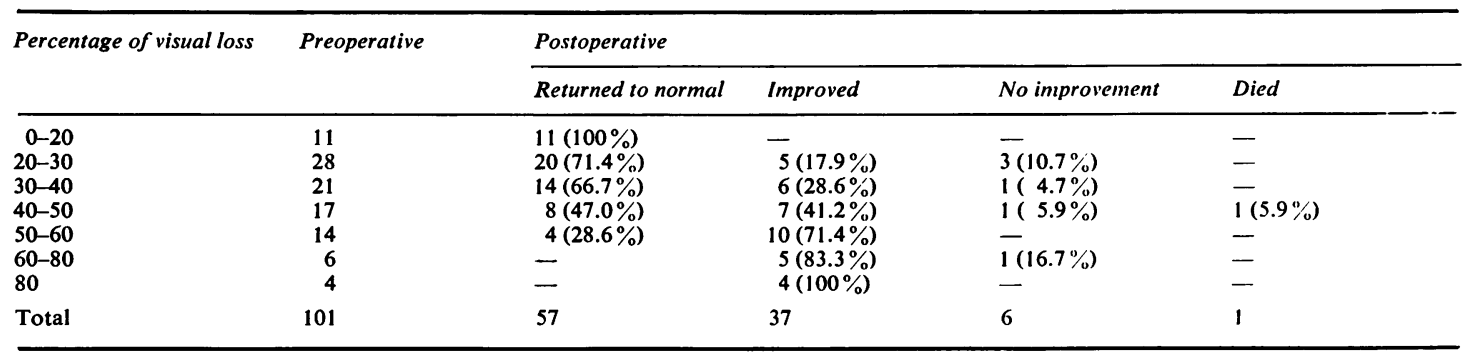

subsequently developing signs of chiasmal compression. Six patients were acromegalic and also developed visual loss.

Six patients were admitted with a history and signs suggesting pituitary apoplexy, five of them were transferred from other hospitals where they had been diagnosed and treated with dexamethasone and, as there was only a limited improvement in vision, then referred for surgical treatment. All five patients were in satisfactory general condition. One patient (a woman of 46 years), was admitted directly from home after the sudden development of blindness and deteriorating level of consciousness. She was found to have a bilateral ophthalmoplegia, complete blindness in one eye, and visual acuity reduced to perception of finger movement in the other. Treatment with dexamethasone resulted in considerable improvement in her general condition and some improvement in her vision.

Two other patients had a dual pathology. A man aged 45 years who was admitted with signs of raised intracranial pressure and a left homonymous hemianopia, showed an eroded sella turcica on plain skull radiographs. Carotid angiography showed the presence of a right temporal tumour and a coincidental pituitary fossa lesion with suprasellar extension. A right temporal lobectomy and subtotal excision of an ependymoma was performed. This was followed by deep x-ray therapy. One year later he returned because of deterioration in his vision and had a second operation for

Table 7 Length of history and sella turcica changes

\begin{tabular}{lllll}
\hline $\begin{array}{l}\text { Length } \\
\text { of } \\
\text { history } \\
(y r)\end{array}$ & NAD & Moderate changes & $\begin{array}{l}\text { Gross destruction } \\
\text { or sphenoidal } \\
\text { sinus involvement }\end{array}$ & $\begin{array}{l}\text { Number of } \\
\text { cases }\end{array}$ \\
\hline $0-1$ & 3 & $25(78.1 \%)$ & $4(21.9 \%)$ & 32 \\
$1-2$ & - & $23(95.8 \%)$ & $1(4.2 \%)$ & 24 \\
$2-4$ & - & $17(80.5 \%)$ & $4(19.5 \%)$ & 21 \\
$4-6$ & - & $9(90 \%)$ & $1(10 \%)$ & 10 \\
$6-7$ & - & $7(50 \%)$ & $7(50 \%)$ & 14 \\
Total & 3 & $81(80.2 \%)$ & $17(16.8 \%)$ & $101(100 \%)$ \\
& $(2.97 \%)$ & & \\
\hline
\end{tabular}

pituitary tumour. The second case was a female aged 44 years with a 20 year history of hormonal dysfunction, one year history of proptosis of the right eye and diplopia, and a two month history of right temporal hemianopia and reduction of visual acuity in both eyes (35\% visual loss). Investigations showed signs of a pituitary fossa tumour with significant suprasellar extension and coincidental right orbital angioma. Both lesions were excised at the same operation.

\section{INVESTIGATIONS}

All our patients had full endocrinological assessment elsewhcre and the figures have not been analysed here.

Skull radiographs were evaluated in all 1019 cases. Moderate destructive changes were found in 8 81 patients; gross destruction of the sella with or without signs of sphenoidal sinus involvement was found in 17 patients. Normal skull radiographs? were found in three cases (all suffered from visual loss and had substantial suprasellar extension). Eleven patients were originally treated for amenorrhoea or infertility without having plain skull radiographs, or with radiology only at a later stage when visual loss was already established. The correlation between the duration of symptoms and skull radiographic changes is shown in Table 7 . It is interesting to note that all patients with a normal sella had a short history (up to one year).

A CAT scan was performed in 29 cases (transverse cuts only) and in all cases enhancement with iothalamate was carried out. In 28 patients there were signs of a suprasellar or intrasellar mass; one examination was negative. Of 28 positive tests three suggested the presence of a craniopharyngioma: in two others the diagnosis was uncertain.

Pneumoencephalography was carried out on 96 patients but not on those who were admitted as an emergency with signs of pituitary apoplexy. We evaluated suprasellar extension, its size and direction and also the degree of involvement of the third ventricle, lateral ventricles, carotid artery 
cistern, and chiasmatic cistern. Seventy-six patients $(79 \%)$ had a central extension and 20 patients $(21 \%)$ had a lateral, posterior, or subfrontal extension of the tumour. The suprasellar extension was classified as small (up to $10 \mathrm{~mm}$ above the sphenoidal plane) in 11 cases $(11.4 \%)$, medium-sized (up to $25 \mathrm{~mm}$ ) in 50 cases $(52.1 \%$ ), and large (over $20 \mathrm{~mm}$ ) in 35 cases $(36.5 \%$ ).

Lateral ventricular enlargement was found in 11 cases $(11.4 \%)$; in two cases $(2.1 \%)$ the patient suffered from raised pressure hydrocephalus probably because of obliteration of the basal cisterns, and in nine patients $(9.3 \%)$ ventricular dilatation was probably caused by old age and atrophy (Table 8).

Table 8 Pneumoencephalographic findings in 96 patients

\begin{tabular}{|c|c|c|}
\hline Suprasellar extension & Size & $\begin{array}{l}\text { Number } \\
\text { of cases }\end{array}$ \\
\hline 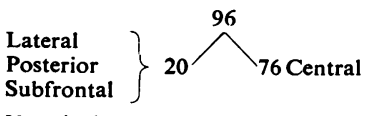 & $\begin{array}{l}\text { Small }-10 \mathrm{~mm} \\
\text { Medium }-25 \mathrm{~mm} \\
\text { Large }>25 \mathrm{~mm}\end{array}$ & $\begin{array}{l}11(11.4 \%) \\
50(52.1 \%) \\
35(36.5 \%)\end{array}$ \\
\hline Ventricular enlargement & & $11(11.4 \%)$ \\
\hline
\end{tabular}

Carotid angiography was performed in 99 cases. However eight patients had angiography performed in other hospitals and the films were not available for present analysis. In most of the 91 patients examined in our hospital, bilateral angiography was carried out unless good filling of the intracavernous portion of the opposite carotid artery was obtained with cross compression. Magnification and subtraction techniques were in common use. The angiographic findings are presented in Table 9. There were a small number of negative studies-five cases $(5.5 \%)$, and a considerable number with incidental aneurysm in near contact with the tumour-six cases $(6.5 \%)$. Involvement of internal carotid arteries was the most consistent feature, present in 74 cases $(81.3 \%)$. Elevation of

Table 9 Angiographic findings in 91 patients

\begin{tabular}{llc}
\hline Finding & Number of cases & $\%$ \\
\hline ICA involvement & 74 & 81.3 \\
ACA involvement & 64 & 70.3 \\
Basilar artery involvement & 1 & 13.1 \\
Tumour blush & 12 & 6.5 \\
Aneurysms & 6 & \\
Hydrocephalus & 1 & \\
Arterial spasm & 1 & 5.5 \\
Tumours elsewhere & 2 & \\
No abnormality & 5 & \\
\hline
\end{tabular}

ICA = internal carotid artery.

$\mathbf{A C A}=$ anterior cerebral artery. anterior cerebral arteries was found in 64 cases (70.3\%). A large tumour blush was detected in 12 cases $(13.1 \%)$.

\section{MANAGEMENT}

All patients had preoperative endocrine assessment carried out by endocrinologists elsewhere. Full ophthalmological assessment was carried out in our department, all invasive investigations were performed under steroid cover, and the patients received a loading dose of dexamethasone the day before operation.

Operation was carried out under general anaesthesia with controlled respiration, using almost exclusively a small right frontal craniotomy flap. Particular care was taken to decompress both optic nerves and the chiasm and to free them from the capsule, and the redundant capsule was then excised as far as possible, the posterior remnants of the capsule being pulled down into the sella. The optic chiasma was never separated from the third ventricle and the pituitary stalk was preserved when possible.

The general policy was to carry out as radical an excision as might be. Eighty-eight cases were treated with radical excision of the tumour. Partial excision was performed in 12 cases and extensive tumour biopsy in one case. In all cases where partial excision or tumour biopsy only was carried out, the tumour was either very hard and vascular, adherent to the vessels or inaccessible, and more radical surgery judged dangerous. However, adequate decompression of the optic nerves and chiasma was always ensured. In one case where only biopsy was carried out the tumour was very large and extending posteriorly with a prefixed chiasma. As the preoperative visual loss did not exceed $20 \%$ it was decided to rely on x-ray therapy only. After operation, patients were treated with dexamethasone $4 \mathrm{mg}$ four times a day, in decreasing doses and then established on supplementary cortisone acetate $25 \mathrm{mg}$ in the morning and 12.5 $\mathrm{mg}$ in the evening. Fluid balance was monitored, urine volume and specific gravity were recorded four hourly, and urine osmolarity daily. For the first few days after operation blood electrolytes and osmolality were also examined daily. Treatment with antidiuretics was avoided during the first postoperative 24 hours whenever possible. The situation was then reappraised and if required, DDAVP or other antidiuretic was given as necessary. During the postoperative period the patients were particularly observed for signs of inappropriate secretion of antidiuretic hormone and overhydration to prevent the development of localised brain oedema of the retracted hemisphere. Having 
recovered from the postoperative period, patients were referred for deep x-ray therapy. Most patients were fit to start irradiation approximately three weeks after craniotomy, when their flap was well healed.

Treatment, mostly on an outpatient basis was given three times a week for four weeks and consisted of 12 treatments, a dose of 3600 rads maximum and 3250 rads minimum being given to the tumour volume. This is equivalent to a dose of 4000 rads if treatment is given on five days a week for four weeks.

After completion of deep x-ray therapy patients were referred to endocrinologists for postoperative endocrine assessment and replacement therapy. All patients were seen in the first three months after the operation, in the next six months, and then followed up at yearly intervals, remaining under endocrinological and ophthalmological supervision also.

\section{Results}

The average length of stay in our unit was 10 days. All patients survived the first postoperative period, but one died a week later, a woman of 78 years of age, with rapidly deteriorating vision. Although she was hypertensive $(220 / 120 \mathrm{mmHg})$ her preoperative general condition was satisfactory. Studies showed a moderately large tumour which was partially removed. Postoperatively she was well, but on the seventh day she developed pulmonary embolism and died shortly afterwards. The operative mortality is thus $0.99 \%$.

Three patients developed early postoperative CSF rhinorrhoea and were reoperated a few days after the original surgery. The sella turcica was packed with muscle and the leak stopped.

Of 100 surviving cases the early operative result was evaluated in 53 cases as "excellent," in 35 patients as "good," and in 12 cases as "satisfactory." All patients classified as "excellent" or "good" returned to preoperative employment or activities. Ophthalmological evaluation showed rapid visual improvement after the operation and further improvement in the next six months. Figure 2 shows the result of early postoperative evaluation of visual loss and later follow-up. The number of patients with no visual loss increased from 25 in the early postoperative period to 57 six months after operation. There was a tendency for improvement of vision in all groups of visual disability. However, the largest number of patients who returned to normal in the next six months came from the group with relatively minor postoperative visual loss (5 to $20 \%$ ). The final post-

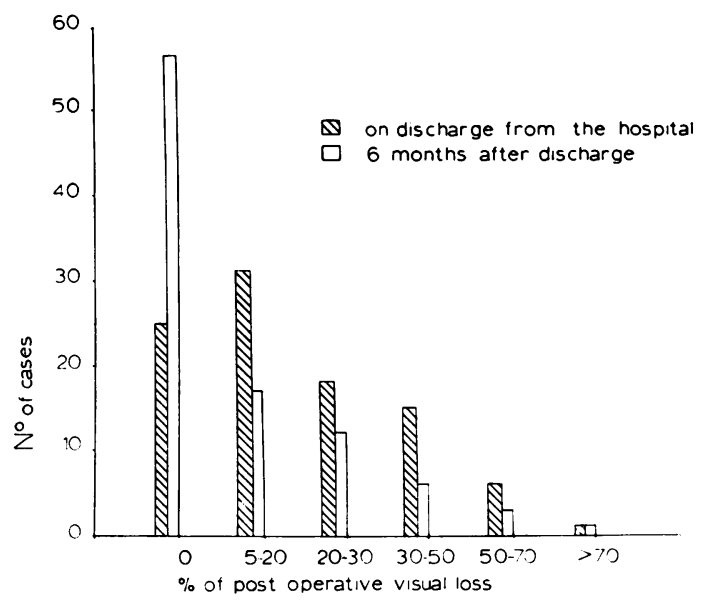

Fig. 2 Evaluation of percentage of postoperative visual loss of patients on day of discharge from hospital and approximately six months later.

operative assessment of visual defect showed that out of the 100 patients 57 had regained normal vision, 37 had considerable improvement of vision, and only six patients remained unimproved.

We have correlated the postoperative visual recovery with (1) the extent of preoperative visual loss, (2) the duration of preoperative visual symptoms, (3) the age of the patients, and (4) the size of the tumour.

Table 6 and Fig. 3 summarise the correlations between the percentage of preoperative visual loss and postoperative recovery. There was $100 \%$ recovery in the group (11 cases) when preoperative visual loss was below $20 \%$ with preoperative visual loss between 20 and $30 \%$ total recovery was reduced to $71.4 \%$ ( 20 cases), and in the next group where preoperative visual loss was between 30 and $40 \%$ full vision recovered in $66.7 \%$ (14 cases). No case showed full visual recovery where preoperative visual loss was $60 \%$ or above.

Correlation between the duration of visual symptoms preoperatively and final postoperative visual recovery showed that in the group of patients who had a full recovery the mean length of symptoms was no longer than around one year; patients who achieved progressively less visual recovery gave a correspondingly longer mean length of history (Fig. 4).

The average age of patients with full visual recovery was 45.7 years (SD 10.6) and of patients with partial or no recovery was 52.7 years (SD 8.96). More detailed analysis of the age groups showed proportional decrease in full visual recovery in each consecutive decade over 40 years. Over 60 years this reduction was still sharper. 


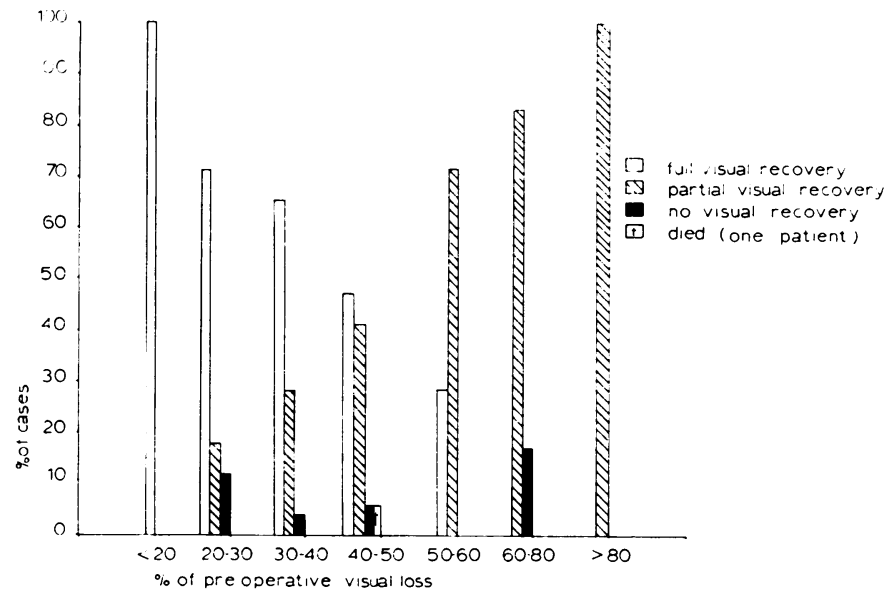

Fig. 3 Analysis of degree of postoperative visual recovery in relation to percentage of preoperative visual loss.

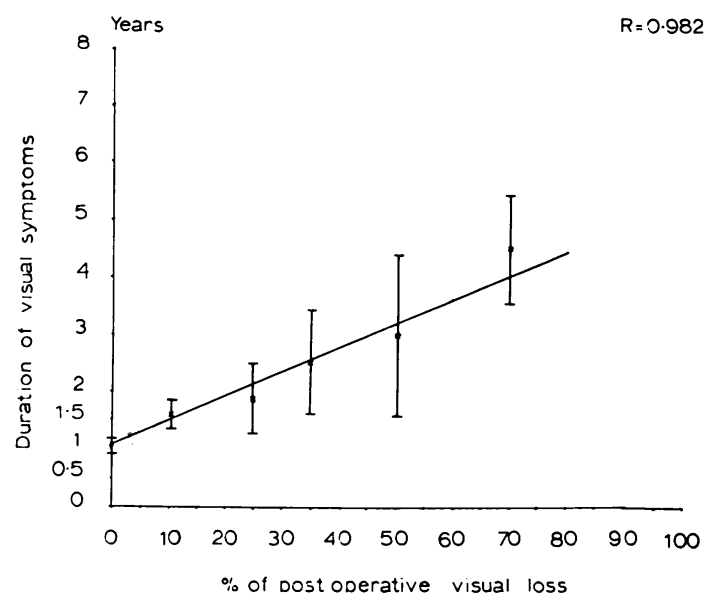

Fig. 4 Mean values of preoperative duration of visual symptoms in relation to percentage of postoperative visual loss.

Further correlations with the size of the tumour showed that in patients with a large tumour there was a much better rate of full recovery of vision in the under 40s and a consequent drop in recovery rate in each subsequent decade. The influence of age on both those patients with small and medium tumours was not significant until the age of 60 years. Of these only $20 \%$ recovered fully and $80 \%$ partially. In summary, it is possible to say that age influences the completeness of visual recovery in patients with large tumours but does not contribute much either way in cases of small and medium tumours up to the age of 60 years (Table 10). There was no correlation between the size of tumour and preoperative visual deficit. Further, there was no obvious influence of the length of the history on the size of suprasellar extension.

ENDOCRINOLOGICAL ASSESSMENT

Postoperative diabetes insipidus was noted in 49 patients; in 36 patients it improved in the early postoperative period, but in 13 cases it remained for a more prolonged period. Three of those, however, are still within the first six months after the operation. In three cases who suffered from preoperative diabetes insipidus polyuria was arrested. All our patients needed postoperative hormonal replacement. However, further endocrinological assessment showed that two cases had regained normal endocrine function.

Histological examination of the tumour removed during operation showed that out of a total number of 101 cases, 80 patients suffered from chromophobe adenoma, 12 patients had a mixed type adenoma, seven patients had an eosinophil adenoma, and two patients had a basophil adenoma.

\section{FOLLOW-UP}

Out of 100 patients who survived the operation we have up-to-date follow-up in 97 patients. Three patients were followed for only a limited period, one for 10 months and two others for a period of one year with an outcome evaluated as good in one patient and satisfactory in two patients. Contact with them has been lost as two have left the area and one left the country. Two further patients have died in later follow-up. The first was a man of 63 years whose general condition before operation was poor. He survived the operation, made a satisfactory recovery, but five months later died suddenly at home, either from coronary artery thrombosis or pulmonary embolism; unfortunately 
Table 10 Age of patients, size of tumour, and degree of postoperative visual recovery

\begin{tabular}{|c|c|c|c|c|c|}
\hline Age groups (yr) & $<40$ & $40-50$ & $50-60$ & $60>$ & \\
\hline Vision returned to normal & $\begin{array}{l}\text { (12) } 75 \% \\
66.7 \%(6) \\
\text { (6) } 85.7 \%\end{array}$ & $\begin{array}{l}\text { (24) } 68.6 \% \\
73.9 \%(17) \\
\text { (7) } 58.3 \%\end{array}$ & $\begin{array}{l}\text { (19) } 50 \% \\
60 \%(15) \\
\text { (4) } 30.8 \%\end{array}$ & $\begin{array}{l}\text { (2) } 6.7 \% \\
20 \%(15) \\
(-) 0 \%\end{array}$ & $\begin{array}{l}=57 \\
\mathbf{M}+\mathbf{S} \\
\mathrm{L}\end{array}$ \\
\hline Vision improved or no change & $\begin{array}{l}\text { (4) } 25 \% \\
33.3 \%(3) \\
\text { (1) } 14.3 \%\end{array}$ & $\begin{array}{l}\text { (11) } 31.4 \% \\
26.1 \%(6) \\
\text { (5) } 41.7 \%\end{array}$ & $\begin{array}{l}\text { (19) } 50 \% \\
40 \%(10) \\
\text { (9) } 69.2 \%\end{array}$ & $\begin{array}{l}\text { (10) } 83.3 \% \\
80 \%(8) \\
(2) 100 \%\end{array}$ & $\begin{array}{l}=44 \\
M \\
L\end{array}$ \\
\hline
\end{tabular}

$\mathrm{S}=$ small suprasellar extension; $\mathrm{M}=$ medium suprasellar extension; $\mathrm{L}=$ large suprasellar extension

no postmortem examination was carried out. The second patient was a man of 46 years who had an invasive chromophobe adenoma infiltrating the sphenoidal sinus. The tumour was partially removed and treatment was completed with a course of $\mathrm{x}$-ray therapy. The outcome was evaluated as good and the patient returned to his previous employment. Five years later, however, he developed CSF rhinorrhoea, and was operated on again, no sign of intracranial recurrence was found, and the sella turcica was empty. The sella was filled with muscle and this arrested the rhinorrhoea; he was discharged home but one year later developed meningitis and died elsewhere.

Of the remaining 95 cases, 21 patients were followed for a period of eight years or over, 34 patients for between four and eight years, and a further 40 patients for from six months to four years. The exact numbers, are shown in Table 11 . Up to now we have no case of recurrence.

The quality of postoperative survival and quality of life as reported during the late follow-up are shown in Table 12. The quality of life has remained the same in all 53 cases whose outcome of treatment in early follow-up was classified as excellent (two of the patients have not yet had a late followup). Of the group of 35 patients where the outcome of treatment was classified as good 28 maintained good quality of life. Three patients developed late postoperative rhinorrhoea, two of them had radical excision of the tumour and one partial excision

Table 11 Length of postoperative follow-up

\begin{tabular}{ll}
\hline Follow-up $(y r)$ & Number of cases \\
\hline $0-1$ & 20 \\
$1-2$ & 14 \\
$2-4$ & 6 \\
$4-6$ & 22 \\
$6-8$ & 12 \\
$8>$ & 21 \\
Total & 95 \\
\hline
\end{tabular}

One patient was followed only for $\mathbf{1 0}$ months and two for only a year because they left the area.

One died six years after operation from meningitis.

One died four months after operation from coronary thrombosis.

There was one operative death from pulmonary embolism.
Table 12 Results of follow-up

\begin{tabular}{lrllll}
\hline Outcome of surgery & $\begin{array}{l}\text { Uneventful Early } \\
\text { course of } \\
\text { life }\end{array}$ & $\begin{array}{l}\text { lost- } \\
\text { poperative } \\
\text { oper }\end{array}$ & $\begin{array}{l}\text { Not } \\
\text { followed- } \\
\text { up }\end{array}$ & Complications \\
\hline Excellent & 53 & 51 & 2 & - & - \\
Good & 35 & 29 & 2 & 1 & $3(1+) \mathrm{R}$ \\
Satisfactory & 12 & 8 & 1 & 2 & $1+\mathrm{CR}$ \\
Dead & 1 & - & - & - & - \\
Total & 101 & 88 & 5 & 3 & 4 \\
\hline
\end{tabular}

$\mathbf{R}=$ rhinorrhoea $; \mathbf{C R}=$ cardiorespiratory $;+=$ death.

of the tumour, and all had received x-ray therapy. Two were reoperated intracranially and one transsphenoidally. In none of the three cases was there evidence of tumour recurrence, and the CSF leak was stopped successfully. One patient, however, developed meningitis a year later and died (see above). Two patients are in early postoperative state and one patient has been lost to follow-up. In the group of 12 patients where the outcome of treatment was classified as satisfactory, eight remained the same, one died five months after discharge from a cardiorespiratory problem, one is still in the early postoperative period, and two patients have no recent follow-up.

Analysis of the follow-up of 13 cases where tumours were either partially removed or biopsied showed that one died in the postoperative period, 12 patients survived the operation and had deep $\mathrm{x}$-ray therapy. One patient was not seen for five years (he had emigrated). One patient died six years after the operation from meningitis (see above), the remaining 10 patients are alive. In five cases the quality of life could be described as excellent, in four as good, and in one as satisfactory. Two patients had been followed for a period of over eight years and the remaining eight patients for a period of one to six years.

\section{Discussion}

Recent advances in diagnostic techniques and microneurosurgery have not only increased the operability of patients with large tumours but have enhanced the extent of excision of the tumour. 
In such elective surgery the maximum preoperative information is desirable. In most of our patients, both arteriography and pneumoencephalography were carried out; we believe both to be necessary tests. Plain skull radiography and pneumoencephalography in association with a suggestive clinical picture, may not always be adequate. The information added by CAT scan is also partial, but nevertheless valuable in the preliminary selection of patients. Modern angiography, with magnification and subtraction techniques, gives additional information such as the degree of involvement of major vessels, the extent of cavernous sinus extension, and presence of coexisting pathology such as aneurysms in the vicinity of the tumour (Jakubowski and Kendall, 1978). If radical excision of the tumour is deemed advisable, as we believe, such information is essential if operative mortality is to be reduced below the figure of $1 \%$. We have not found that the techniques of modern carotid angiography add to the morbidity of management.

We favour radical excision of pituitary adenomas both to reduce the rate of recurrence and to ensure more adequate decompression of optic nerves, optic chiasma, and vessels, with maximal improvement in postoperative visual results. Preservation of the vascular supply to the chiasma and optic nerves is as important as the decompression itself. We believe that it is unwise to dissect the chiasma from the floor of the third ventricle in the presence of tumour compression from below. The disturbance of both superior and inferior chiasmatic plexuses may be too traumatic in some circumstances and impair the operative result. The transfrontal approach between the optic nerves is very satisfactory in combination with microsurgical technique, and seems in general less hazardous than the route lateral to the optic nerve advocated by von Alphen (1975). In a minority of cases extensive radical removal is not possible, but the so-called partial removal of tumour with suprasellar extension to preserve some remnants of the normal pituitary tissue seems, generally, without merit.

While the importance of postoperative $x$-ray therapy may be questioned, we believe that modern well-balanced $\mathrm{x}$-ray treatment is essential, even after the most radical removal of the tumour. It seems that the advantages of x-ray treatment, because of the extremely high x-ray sensitivity of the tumour, outweigh the theoretical disadvantage of disturbance of the body immune mechanism, unlikely in selective radiation. The absence of recurrence in the present series supports this view, although only $60 \%$ of the present cases have a follow-up period of over four years.

Adequate hormone replacement therapy plays an important role in the quality of postoperative results and perhaps decreases the rate of recurrence of the tumour. We have found that in extreme states of chiasmatic compression preoperative treatment with dexamethasone improved not only the patients' level of consciousness but also the visual function. Jefferson (1957) has speculated that steroids may shrink the tumour, and perhaps control its growth. Our experience suggests that this may be the case. All postoperative cases should remain under endocrinological supervision.

The main postoperative complication has been CSF rhinorrhoea. This occurred in three cases in the early postoperative period and in all cases there was considerable destruction of the sella. We believe that in such circumstances the leak should be closed without delay in view of the risk of meningitis. All our cases were successfully closed with a muscle graft. There seemed no contraindication to $\mathrm{x}$-ray therapy after the leak was stopped. Another complication was postoperative diabetes insipidus $(49 \%)$. More often than not it was transient and easily controllable with drugs. However, it has remained permanent in some $13 \%$ of our cases. While this may be related to the radical nature of the surgery, an additional three patients with chromophobe adenoma who suffered from diabetes insipidus preoperatively showed arrest of polyuria after radical removal of the tumour. Careful postoperative monitoring of fluid balance is of great importance. Overhydrated patients after pituitary surgery are vulnerable to unilateral swelling of the retracted frontal lobe which may imitate mass compression and lead to unnecessary re-exploration. We feel that treatment with antidiuretics should, therefore, be restrained during the first 24 hours if possible. After a baseline has been established, one can easily correct the negative fluid balance.

The mortality rate (Table 1) has varied in various series and in some it is also influenced by the selection of patients. The size of the tumour and its possible recurrent nature are factors generally thought to produce high surgical mortality. The loss of one patient in our series (around $1 \%$ mortality) was the result of pulmonary embolism, a well-recognised and unpredictable surgical complication. The patient was in the advanced age group, and in retrospect it might have been wiser to rely on radiotherapy and hormone replacement. If the incidental complications leading to postoperative death are not considered, the mortality from neurosurgical intervention should be nil, even with 
surgery of those tumours with a moderate or large suprasellar extension. This, unfortunately, does not apply to so-called giant tumours, and the inclusion of 16 such in the present analysis would increase the operative mortality rate to $3.3 \%$. Cases with recurrent tumour originally operated elsewhere, if included, would have no effect on the reported mortality rate.

To evaluate quantitatively the degree of postoperative visual recovery we developed a point scoring system. While by no means fully comprehensive it covers most aspects and is simple. By its use we were able to compare preoperative visual loss and postoperative visual recovery. An immediate dramatic postoperative improvement resulted in 25 patients regaining normal vision early. Further follow-up showed that within the next six months the number of patients restored to full vision had increased to 57 , and others (with the exception of six who remained the same) had further considerable improvement. From the analysis of our cases it appeared that the degree of postoperative visual recovery depended on various factors. It was much influenced by the degree of preoperative visual loss, indeed in all cases where preoperative visual loss is below $20 \%$ postoperative recovery should be complete. However, when preoperative visual loss is over $60 \%$ full recovery of vision is unlikely. A further important factor influencing the postoperative visual recovery in this series was the duration of preoperative visual symptoms. The average length of history for the patient who had full postoperative visual recovery was about one year. The importance of early referral of such patients to surgery is, therefore, clear. Neither the age nor the size of the tumour was a direct factor in the degree of postoperative visual recovery in patients below age 60 years in tumours with small and medium suprasellar extensions. However, full visual recovery was correlated with age in patients with large tumours.

We were unable to establish significant correlation between the degree of preoperative visual loss, the age of the patient, and the size of the tumour. This confirms Dawson's and Morello's observations that the preoperative integrity of the vascular supply to the chiasma and optic nerves is of primary importance.

The major variable apart from surgery which could improve the degree of all postoperative visual recovery was the duration of visual symptoms. Analysis of the pattern or referral of patients showed that most of our patients $(87 \%)$ were referred by neurologists, of ten after endocrine or ophthalmic referral. Unexplained loss of visual acuity without obvious peripheral visual field loss was often not regarded as an indication for further investigations by the patient's general practitioner or optician. Some patients were treated for amenorrhoea and infertility by their family doctor or gynaecologist for a substantial length of time without ophthalmological evaluation and without skull radiograph at the beginning of therapy. Visual examination of the patient with a longstanding endocrine disturbance was not always performed with adequate frequency and skill, particularly the assessment of central fields. Fortyfour of our patients had a history of visual complaint over one year, and 27 of them had a history varying between two years and 10 years. This seems unacceptable by present day standards. The frequency of pituitary fossa tumours with suprasellar extension should be recalled widely in ophthalmic, gynaecological, and general practice. From the moment the patient was referred to a neurologist or neurosurgeon there was no further delay in treatment.

We wish to thank Professor G. M. Besser of St Bartholomew's Hospital, London, and Dr P. H. Sonksen and Dr Clara Lowy of St Thomas' Hospital, London for the endocrinological management of our patients. We also wish to thank Dr D. Collins of St Thomas' Hospital, London, for x-ray therapy of most of our patients. We are indebted to colleagues from the National Hospital, Queen Square and the Maida Vale Hospital, London, for referring their patients to us.

\section{References}

Alphen, von H. A. M. (1975). Microsurgical frontotemporal approach to pituitary adenomas with extrasellar extension. Clinical Neurology and Neurosurgery, 78, 246-256.

Arumugasany, N., Lestina, F. A., and Bucy, P. C. (1971). Treatment of pituitary adenomas: a study of 66 cases. Medical Journal of Malaya, 26, 3-14.

Bakay, L. (1950). The results of 300 pituitary adenoma operations (Professor Herbert Olivecrona's series). Journal of Neurosurgery, 7, 240-255.

Baker, G. S. (1960). Treatment of pituitary adenomas. Archives of Surgery (Chicago), 81, 842-846.

Dawson, B. H. (1958). The blood vessels of the human optic chiasma and their relation to those of the hypophysis and hypothalamus. Brain, 81, 207-217.

Elkington, S. G., and McKissock, W. (1967). Pituitary adenoma. Results of combined surgical and radiotherapeutic treatment of 260 patients. British Medical Journal, 1, 263-266.

Guillaume, J., and Caron, J. P. (1958). Remarques cliniques et chirurgicales relatives aux adenomas hypophysoires. Neurochirurgie, 4, 338-343. 
Horrax, G., Hare, J. F., Poppen, J. L., Hurxthal, L. M., and Younghusband, D. Z. (1952). Chromophobe pituitary tumours II. Treatment. Journal of Clinical Endocrinology and Metabolism, 12, 631641.

Jakubowski, J., and Kendall, B. (1978). Coincidental aneurysms with tumours of pituitary origin. Journal of Neurology, Neurosurgery, and Psychiatry, 41, 972-979.

Jefferson, A. (1957). Some clinical features of the pituitary chromophobe adenomata and of the Rathke pouch cysts. Annals of the Royal College of Surgeons of England, 21, 358-381.

Jefferson, A. (1969). Chromophobe pituitary adenomata. The size of the suprasellar portion in relation to the safety of operation. Journal of Neurology, Neurosurgery, and Psychiatry, 32, 632.

Jefferson, A. (1978). Transcranial operation and radiotherapy of pituitary adenomas. In Treatment of Pituitary Adenomas: a European Workshop. Edited by R. Fahlbusch and K. V. Werder. Georg Thieme Verlag: Stuttgart.

Jefferson, G. (1940). Extrasellar extensions of pituitary adenomas. President's address. Proceedings of the Royal Society of Medicine, 33, 433-458.

Krayenbühl, H. (1961). Hypophyseal adenomas and craniopharyngiomas. Abstracts and descriptions of the scientific program of the 2nd International Congress of Neurological Surgery. Excerpta Medica, International Congress Series, 36, E10-E12.

Kunc, Z. (1973). Problemy chirurgie chromophobnich adenoma hypofyzy. Ceskoslovenska Neurologie a Neurochirurgie, 36, 349-356.
Kunicki, A., Mądraszkiewicz, E., and Szwagrzyk, E. (1975). Postepowanie chirurgiczne i wyniki leczenia w 114 przypadkach gruczolaka przysadki mózgowej. Neurologia Neurochirurgia Polska, 25, 503-509.

Mogensen, E. F. (1957). Chromophobe adenoma of pituitary gland: a follow-up study on 60 surgical patients with special reference to endocrine disturbances. Acta Endocrinologica (Copenhagen), 24, 135-152.

Morello, G., and Frera, C. (1966). Visual damage after removal of hypophyseal adenomas: possible importance of vascular disturbances of optic nerves and chiasma. Acta Neurochirurgica, 15, 1-10.

Obrador, A. S. (1961). Adenomas of the pituitary based on a neurosurgical experience of 65 operated patients. Revista Clinica Española, 81, 396-440.

Rand, C. W. (1957). Notes on pituitary tumours: including suggestions of others, and personal experiences in 100 cases. Clinical Neurosurgery, 3, 1-58.

Ray, B. S., and Patterson, R. H. (1971). Surgical experience with chromophobe adenomas of pituitary gland. Journal of Neurosurgery, 34, 726-729.

Stern, W. E., and Batzdorf, U. (1970). Intracranial removal of pituitary adenomas. An evolution of varying degrees of excision from partial to total. Journal of Neurosurgery, 33, 564-573.

Tönnis, W., Oberdisse, K., and Weber, E. (1953). Bericht uber 264 operierte hypophysen-adenome. Acta Neurochirurgica, 3, 113-130.

Wirth, F. P., Schwartz, H. G., and Schwetschenae, P. R. (1974). Pituitary adenomas: factors in treatment. Clinical Neurosurgery, 21, 8-25. 